\title{
Countable compactness in generalized L-topological spaces
}

\author{
Shi-Zhong Bai ${ }^{1, \mathrm{a}}$, Yi Shi ${ }^{1, \mathrm{~b}}$ \\ ${ }^{1}$ School of Mathematics and Computational Science, Wuyi \\ University, Guangdong, China \\ ashizhongbai@aliyun.com, symathematics@163.com
}

\begin{abstract}
.
In this paper, the generalized countable L-compact sets and generalized Lindelöf sets are introduced in generalized L-topological spaces, based on the notion of generalized L-compactness. They are described by cover form and finite intersection property. They are preserved under generalized L-continuous mapping, inherited for L-closed subsets, and finitely additive. And an L-subset is generalized L-compact if and only if it is generalized Lindelöf and generalized countably L-compact.
\end{abstract}

Keywords: generalized L-topology, generalized countable L-compactness,generalized Lindelöf set

\section{Introduction and Preliminaries}

In[2],Bai introduced the concept of generalized L-topological spaces, and studied the basic concepts and basic properties in generalized L-topological spaces. Following the lines of [2], in [3], Bai introduced generalized L-compactness. In this paper, our aim is to continue the research of generalized countable L-compact sets and generalized Lindelöf sets in generalized L-topological spaces.

Throughout this paper, $\left(L, \vee, \wedge,{ }^{\prime}\right)$ is a completely distributive De Morgan algebra and $X$ is a nonempty set. $L^{X}$ is the set of all L-fuzzy sets on $X$. The smallest element and the largest element of $L^{X}$ will be denoted by 0 and 1 respectively.The set of non-unit prime elements[4] in $L$ is denoted by $\operatorname{pr}(L)$. The set of nonzero co-prime elements[4] in $L$ and $L^{X}$ is denoted by 
$M(L)$ and $M^{*}(L)$ respectively. Clearly, $r \in \operatorname{pr}(L)$ iff $r^{\prime} \in M(L)$.The greatest minimal family of $a$ in $\mathrm{L}$ is denoted by $\beta(a)$.The greatest maximal family of $a$ in $\mathrm{L}$ is denoted by $\alpha(a)$ [5,8]. Moreover for $a$ in L, define $\beta^{*}(a)=\beta(a) \cap M(L)$ and $\alpha^{*}(a)=\alpha(a) \cap \operatorname{pr}(L)$.For each $\psi \subset L$,we define $\psi^{\prime}=\left\{A^{\prime}: A \in \psi\right\}$. For $r \in L, \varepsilon_{r}(A)=\{x \in X: A(x) \geq r\}$.

Definition1.1.[2]. Let $L$ be a completely distributive De Morgan algebra, $X$ be a nonempty set and $\delta$ be a collection of subsets of $L^{X}$.Then $\delta$ is called a generalized L-topology (briefly GL-t) on $X$ if $0 \in \delta$ and $G_{i} \in \delta$ for $i \in I \neq \varnothing$ implies $G=\vee_{i \in I} G_{i} \in \delta$. We call the pair $\left(L^{X}, \delta\right)$ a generalized L-topological space (briefly GL-ts) on $X$.The element of $\delta$ are called generalized L-open sets (briefly GL-open sets)and the complements are called generalized L-closed sets (briefly GL-closed sets).We say $\delta$ is strong if $1 \in \delta$.

Definition1.2.[6]. Each mapping $f: X \rightarrow Y$ induces a mapping $f_{L}: X \rightarrow Y$ (called an L-valued Zadeh function or an L-fuzzy mapping or an L-forward power set operator),which is defined by $f_{L} \rightarrow(A)=\vee\{A(x) \mid f(x)=y\} \quad\left(\forall A \in L^{X}, y \in Y\right) \quad$.The $\quad$ right $\quad$ adjoint to $f_{L} \rightarrow$ (called L-backward power set operator)is denoted $f_{L}^{\leftarrow}$ and given by $f_{L}^{\leftarrow}(B)=\vee\left\{A \in L^{X} \mid f_{L}^{\rightarrow}(A) \leq B\right\}=B \circ f\left(\forall B \in L^{X}\right)$.

Definition1.3.[2]. Let( $L^{X}, \delta$ )and( $L^{Y}, \tau$ )be two GL-ts's and $f^{\rightarrow}: L^{X} \rightarrow L^{Y}$ an L-fuzzy mapping. $f^{\rightarrow}$ is called a generalized L-continuous mapping(briefly GL-continuous mapping)if $f^{\leftarrow}(B) \in \delta$ for each $B \in \tau$.

Definition1.4[2]. Let $\left(L^{X}, \delta\right)$ be a GL-ts and $x_{\lambda} \in M^{*}\left(L^{X}\right) . A \in \delta^{\prime}$ is 
called a generalized L- closed remote-neighborhood(briefly GLC-RN)of $x_{\lambda}$,if $x_{\lambda} \leq A . B \in L^{X}$ is called a generalized Lremote-neighborhood(briefly GL-RN)of $x_{\lambda}$ if there is a GLC-RN A of $x_{\lambda}$ such that $B \leq A$.The set of all GLC-RNs(GL-RNs)of $x_{\lambda}$ is denoted by $\eta^{-}\left(x_{\lambda}\right)\left(\eta\left(x_{\lambda}\right)\right)$.

Definition1.5[2]. $\quad \operatorname{Let}\left(L^{X}, \delta \quad\right.$ be a GL-ts, $A \in L^{X}$ and $\alpha \in M(L) . \phi \subset \delta^{\prime}$ is called an $\alpha$-closed -remote neighborhood family of $A$ (briefly $\alpha$-C-RF of $A$ )if for each $x_{\alpha}$ in $A$,there exists a $P \in \phi$ such that $P \in \eta\left(x_{\alpha}\right)$. $\phi$ is called an $\alpha^{-}$-C-RF of $A$.

Definition1.6[2]. Let $\left(L^{X}, \delta\right.$ )be a GL-ts and $A \in L^{X} . A$ is called generalized L-compact(briefly GL-compact)if every $\alpha$-C-RF $\phi$ of $A$ has a finite subfamily which is an $\alpha^{-}$-C-RF of $A(\alpha \in M(L))$. ( $L^{X}, \delta$ )is called GL-compact if $1_{X}$ is GL-compact.

\section{Generalized countable L-compactness}

Definition2.1. Let $\left(L^{X}, \delta\right)$ be a GL-ts and $A \in L^{X} . A$ is called generalized countably L-compact if every countable $\alpha-\mathrm{C}-\mathrm{RF} \phi$ of $A$ has a finite subfamily which is an $\alpha^{-}$-C-RF of $A(\alpha \in M(L))$. ( $L^{X}$,

$\delta$ )is called generalized countably L-compact if $1_{X}$ is generalized countably L-compact.

From the Definitions 2.1 and 1.6 we immediately obtain the following results.

Corollary 2.2. Every generalized L-compact set is generalized countably L-compact.

Definition2.3. Let $\left(L^{X}, \delta\right)$ be a GL-ts, $A \in L^{X}$ and $r \in \operatorname{pr}(L) \cdot \mu \subset \delta$ is called an $r$-cover of $A$ if for each $x \in \varepsilon_{r^{\prime}}(A)$, there exists an $U \in \mu$ such that $U(x) \leq r . \mu$ is called an $r^{+}$-cover of $A$ if there exists a $t \in \alpha^{*}(r)$ such that $\mu$ is a $t$-cover of $A$.

Theorem2.4. Let $\left(L^{X}, \delta\right)$ be a GL-ts and $r \in \operatorname{pr}(L) . A \in L^{X}$ is generalized countably L-compact if and only if every countable $r$-cover $\mu$ of $A$ has a finite subfamily $v$ which is an $r^{+}$-cover of $A$. 
Proof. Let $A$ be generalized countably L-compact, $\mu$ a countable $r$-cover of $A$ and $r \in \operatorname{pr}(L)$.Put

$\phi=\mu^{\prime}$, then $\phi \subset \delta^{\prime}$ and for each $x \in \varepsilon_{r^{\prime}}(A)$ there exists a $Q=U^{\prime} \in \phi$ such that $U(x) \leq r$, i.e. $r^{\prime} \leq Q(x)$.

Since $r \in \operatorname{pr}(L), r^{\prime} \in M(L)$. By $x_{r^{\prime}} \leq Q$ we have $Q \in \eta\left(x_{r^{\prime}}\right)$, hence $\phi$ is a countable $r^{\prime}$-C-RF of $A$.Since $A$ is generalized countably L-compact,there is a finite subfamily $v$ of $\mu$ such that $\psi=v^{\prime}$

is an $\left(r^{\prime}\right)^{-}$-C-RF of $A$,i.e.for some $t \in \beta^{*}\left(r^{\prime}\right)$ and each $x \in \varepsilon_{r^{\prime}}(A)$,there is a $X(x) \in V$ such that $t \leq V^{\prime}(x)$,equivalently,for some $t^{\prime} \in \alpha^{*}(r)$ and each $x \notin \varepsilon_{r^{\prime}}(A)$,there is a $V(x) \in v$ such that $V(x) \leq t^{\prime}$. Thus $\mu$ has a finite subfamily $v$ which is an $r^{+}$-cover of $A$.

Conversely,suppose every countable $r$-cover $\mu$ of $A$ has a finite subfamily is an $r^{+}$-cover of $A$. Let $\phi$ be a countable $\alpha$-C-RF of $A, \mu=\phi^{\prime}$ and $r=\alpha^{\prime}$.Since $\alpha \in M(L), r \in \operatorname{pr}(L)$. With the method of dual above, it is easily to prove that $\mu$ is a countable $r$-cover of $A$.Suppose $v$ is a finite subfamily of

$\mu$ such that $v$ is an $r^{+}$-cover of $A$.Put $\psi=v^{\prime}$, then $\psi$ is an $\alpha^{-}$-C-RF of $A$.Thus $A$ is generalized countably L-compact.

Definition2.5. Let $\left(L^{X}, \delta\right)$ be a GL-ts, $A \in L^{X}, r \in \operatorname{pr}(L)$ and $\mu \subset L^{X}$.If for every finite subfamily

$v$ of $\mu$ and for each $t \in \alpha^{*}(r)$, there is an $x \in \varepsilon_{t^{\prime}}(A)$ such that $(\wedge v)(x) \geq t^{\prime}$, then we say that $\mu$ has an

$r^{+}$-finite intersection property in $A$.

Theorem2.6. Let $\left(L^{X}, \delta\right)$ be a GL-ts and $r \in \operatorname{pr}(L) . A \in L^{X}$ is generalized countably L-compact if and only if every countable subfamily of GL-closed sets $\mu$ has an $r^{+}$-finite intersection property in $A$, and there is an $x \in \varepsilon_{r^{\prime}}(A)$ such that $(\wedge \mu)(x) \geq r^{\prime}$.

Proof. Let $A$ be generalized countably L-compact.Suppose there is a prime element $e \in \operatorname{pr}(L)$ and

Some countable subfamily of GL-closed sets $\mu$ has an $e^{+}$-finite intersection property in $A$,for each $x$

$\in \varepsilon_{r^{\prime}}(A)$ such that $(\wedge \mu)(x) \geq e^{\prime}$.Then there exists a $B \in \mu$ such that $B(x) \geq e^{\prime}$, i.e. $B^{\prime}(x) \leq e$.This 
shows $\mu^{\prime}$ is a countable $e$-cover of $A$.By the Theorem2.4,there is a finite subfamily $v=\left\{B_{1}, \cdots, B_{n}\right\}$

of $\mu$ such that $v^{\prime}$ is an $e^{+}$-cover of $A$.Hence for some $t \in \alpha^{*}(e)$ and each $x \in \varepsilon_{r^{\prime}}(A)$,there is an $B_{i} \in v$

such / that $\quad B_{i}^{\prime}(x) \leq t \quad$.And/

so $\left(\vee_{i=1}^{n} B_{i}^{\prime}\right)(x) \leq t$,i.e. $(\wedge v)(x)=\left(\wedge_{i=1}^{n} B_{i}\right)(x) \geq t^{\prime}$, which contradicts that $\mu$ has an $e^{+}$-finite intersection property in $A$.

Conversely,let $\mu$ be a countable $r$-cover of $A$ and $r \in \operatorname{pr}(L)$.If none of the finite subfamily $v$ of $\mu$

is $r^{+}$-cover of $A$, then every $t \in \alpha^{*}(r)$ there is an $x \in \varepsilon_{r^{\prime}}(A)$ such that $C(x) \leq t$ for each $C \in v$. And so

$(\vee v)(x) \leq t$,equivalently, $\left(\wedge v^{\prime}\right)(x) \geq t^{\prime}$.This shows that subfamily of GL-closed sets $\mu^{\prime}$ having an

$r^{+}$-finite intersection property in $A$.Hence there is an $x \in \varepsilon_{r^{\prime}}(A)$ such that $\left(\wedge \mu^{\prime}\right)(x) \geq r^{\prime}$,i.e. $(\vee \mu)(x)$

$\leq r$.This implies that $\mu$ is not a countable $r$-cover of $A$, a contradiction.By the

Theorem2.4, $A$ is

generalized countably L-compact.

Theorem2.7. Let $\left(L^{X}, \delta\right)$ be a GL-ts and $A, B \in L^{X}$.If $A$ is generalized countably L-compact and

$B \in \delta^{\prime}$,then $A \wedge B$ is generalized countably L-compact.

Proof. Let $\phi \subset \delta^{\prime}$ be a countable $\alpha$-C-RF of $A \wedge B(\alpha \in M(L))$.Then $\phi_{1}=\phi \cup\{B\}$ is a countable $\alpha$-C-RF of $A$.In fact,for each $x_{\alpha} \in B$ then $x_{\alpha} \in A \wedge B$.Hence,there is $P \in \phi \subset \phi_{1}$ such that $P \in \eta\left(x_{\alpha}\right)$.If $x_{\alpha} \notin B$,then $B \in \phi$ and $B \in \eta\left(x_{\alpha}\right)$.Thus, $\phi_{1}$ is indeed a countable $\alpha$-C-RF of $A$.Since $A$ is generalized countably L-compact.there exists an $r \in \beta^{*}(\alpha)$ and finite subfamily $\psi_{1}$ of $\phi_{1}$ such that $\psi_{1}$ is an $r$-C-RF of $A$.Let $\psi=\psi_{1}-\{B\}$, then $\psi$ is a finite subfamily of $\phi$, and $\psi$ is an $r$-C-RF of $A \wedge B$.In fact, $x_{r} \in A \wedge B$,then $x_{r} \in A$,from the definition of $\psi_{1}$, there is $P \in \psi_{1}$, with $P \in \eta_{1}\left(x_{r}\right)$. But $\quad x_{r} \in B \quad$,so $P \neq B$, and thus $P \in \psi_{1}-\{B\}=\psi$.Hence, $A \wedge B$ is generalized countably L-compact.

Theorem2.8. If $A$ and $B$ are generalized countably L-compact in GL-ts 
( $L^{X}, \delta$ ), then $A \vee B$ is generalized countably L-compact.

Proof. This is analogous to the proof of the theorem4.1(2) in [3].

Theorem2.9. Let $\left(L^{X}, \delta\right)$ and $\left(L^{Y}, \tau\right)$ be two GL-ts's, $f^{\rightarrow}: L^{X} \rightarrow L^{Y}$ a GL-continuous mapping and $A$ a generalized countable L-compact set in $\left(L^{X}, \delta\right)$. Then $f^{\rightarrow}(A)$ is generalized countable L-compact in $\left(L^{Y}, \tau\right)$.

Proof. Let $\phi \subset \tau^{\prime}$ be a countable $\alpha$-C-RF of $f^{\rightarrow}(A)$ and $x_{\alpha} \in A(\alpha \in M(L))$.To begin with,let us show that $f^{\leftarrow}(\phi)=\left\{f^{\leftarrow}(P): P \in \phi\right\}$ a countable $\alpha$-C-RF of $A$. Since $f^{\rightarrow}$ is GL-continuous and $x_{\alpha} \in A, f^{\leftarrow}(\phi) \subset \delta^{\prime}$ and $f \rightarrow\left(x_{\alpha}\right)=\left(f^{\rightarrow}(x)\right)_{\alpha} \leq f^{\rightarrow}(A)$. By $\phi$ is a countable $\quad \alpha \quad-\mathrm{C}-\mathrm{RF} \quad$ of $f \rightarrow(A)$, there is a $P \in \phi$ with $P \in \eta\left(\left(f^{\rightarrow}(x)\right)_{\alpha}\right)$,i.e. $\left(f^{\rightarrow}(x)\right)_{\alpha} \leq P$,or,equivalently, $P\left(f^{\rightarrow}(x)\right) \geq \alpha \quad$.By the definition of inverse mapping, $\quad f^{\leftarrow}(P)(x)=P\left(f^{\rightarrow}(x)\right) \geq \alpha \quad$,hence $\quad x_{\alpha} \notin f^{\leftarrow}(P) \quad$,i.e. $f^{\leftarrow}(P) \in \eta\left(x_{\alpha}\right)$.

Therefore $f^{\leftarrow}(\phi)$ is a countable $\alpha$-C-RF of $A$.

Since $A$ is generalized countably L-compact,there exists an $r \in \beta^{*}(\alpha)$ and a finite subfamily $\psi$ of $\phi$ such that $f^{\leftarrow}(\psi)$ is an $r$-C-RF of $A$.Again,by the generalized countable L-compactness of $A$, there exists an $r_{1} \in \beta^{*}(r)$ and a finite subset $\psi_{1}$ of $f^{\leftarrow}(\psi)$ such that $\psi_{1}$ is an $r_{1}$-C-RF of $A$.Obviously we can take $\psi_{1}=f^{\leftarrow}(\psi)$.

Now we will show that $\psi$ is an $r$-C-RF of $f \rightarrow(A)$.Let $y_{r} \leq f^{\rightarrow}(A)$; by the

[8], $r=\sup \left\{\lambda \in L: \exists x \in f^{\leftarrow}(y), A(x) \geq \lambda\right.$ and $\left.\lambda \leq r\right\}$.Since $r_{1} \in \beta^{*}(r)$, we have $r_{1} \in \beta(r)$ and hence there is a $\lambda \in L$ and $x \leq f^{\leftarrow}(y)$ with $A(x) \geq \lambda, \lambda \leq r$, and $\lambda \geq r_{1}$; thus $x_{r_{1}} \leq A$.It follows from $f^{\leftarrow}(\psi)$ s an $r$-C-RF of $A$ that there is a $P \in \psi$ with $f^{\leftarrow}(P) \in \eta\left(x_{r_{1}}\right)$,i.e. $f^{\leftarrow}(P)(x) \geq r_{1}$. Hence $P(y)=$ $P\left(f^{\rightarrow}(x)\right) \nsucceq r_{1} \quad$ and / therefore certainly $P(y) \geq r$,i.e. $P \in \eta\left(y_{r}\right)$.Thus $f^{\rightarrow}(A)$ is generalized countably GL-compact. 


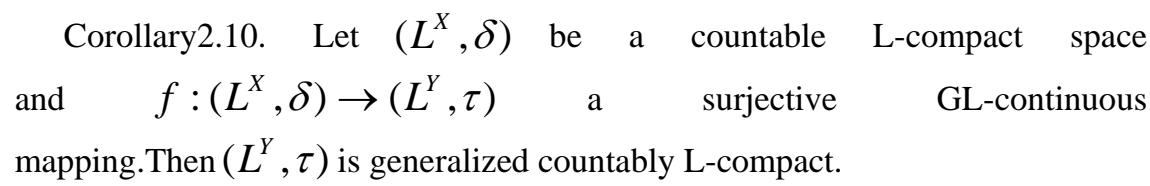

\section{Generalized Lindelöf sets}

Definition3.1. Let $\left(L^{X}, \delta\right)$ be a GL-ts and $A \in L^{X} . A$ is called generalized Lindelöf sets if every $\alpha$-C-RF $\phi$ of $A$ has a countable subfamily which is an $\alpha^{-}$-C-RF $\phi$ of $A(\alpha \in M(L))$. $\left(L^{X}, \delta\right)$ is called generalized Lindelöf space if $1_{X}$ is generalized Lindelöf.

From the Definitions 3.1 and 2.6 we immediately obtain the following results.

Theorem3.2. Let $\left(L^{X}, \delta\right)$ be a GL-ts and $A \in L^{X}$.Then $A$ is generalized L-compact set if and only if $A$ is generalized Lindelöf and generalized countably L-compact.

Analogous to generalized countable L-compactness,we have the following results.

Theorem3.3. Let $\left(L^{X}, \delta\right)$ be a GL-ts and $r \in \operatorname{pr}(L)$.Then $A$ is generalized Lindelöf set if and only if every $r$-cover $\mu$ of $A$ has a countable subfamily $v$ which is an $r^{+}$-cover of $A$.

Theorem3.4. Let $\left(L^{X}, \delta\right)$ be a GL-ts and $A, B \in L^{X}$.If $A$ is generalized Lindelöf set and $B \in \delta^{\prime}$, then

$A \wedge B$ is generalized Lindelöf.

Theorem3.5. If $A$ and $B$ is generalized Lindelöf sets in GL-ts $\left(L^{X}, \delta\right)$, then $A \vee B$ is generalized Lindelöf.

Theorem3.6. Let $\left(L^{X}, \delta\right)$ and $\left(L^{Y}, \tau\right)$ be two GL-ts's, $f^{\rightarrow}: L^{X} \rightarrow L^{Y}$ a GL-continuous mapping and $A$ a generalized Lindelöf set in $\left(L^{X}, \delta\right)$.Then $f^{\rightarrow}(A)$ is generalized Lindelöf in $\left(L^{Y}, \tau\right)$. 
Corollary3.7. Let $\left(L^{X}, \delta\right)$ and $\left(L^{Y}, \tau\right)$ be a generalized Lindelöf set and $\quad f:\left(L^{X}, \delta\right) \rightarrow\left(L^{Y}, \tau\right) \quad$ a $\quad$ surjective $\quad$ GL-continuous mapping.Then $\left(L^{Y}, \tau\right)$ is generalized Lindelöf.

\section{Acknowledgments}

This work is supported by the National Natural Science Foundation of China (No. 11471202), the Natural Science Foundation of Guangdong Province (No.S2012010008833).

\section{References}

[1] S.Z. Bai:Countably compact L-sets,International Journal of Journal of Uncertainty,Fuzziness and Knowledge-based Systems,12(2004),p.115-121

[2] S.Z. Bai:Generalized L-topological spaces,Journal of Intelligent and Fuzzy Systems,in press.

DOI10.3233/IFS-141300.

[3] S.Z. Bai:Generalized compact L-subsets defined by $\alpha$-closed-remote neighborhood family,

Journal of Intelligent and Fuzzy Systems,in press.

[4] G.Gierz:et al.A Compendium of Continuous Lattices,Berlin:Springer,1980.

[5] Y.M.Liu, M.K.Luo:Fuzzy Topology,World Sci.Publishers, Singapore,1998.

[6] S.E.Rodabaugh: Point-set lattice-theoretic topology, Fuzzy Sets and Systems,40(1991),p.297-

345

[7] G.J.Wang:A new fuzzy compactness defined by fuzzy nets,J.Math.Anal.Appl.,94 (1983),p.1-23

[8] G.J.Wang:Theory of L-fuzzy Topological Spaces, Xi'an China:Shaanxi Normal University Press,1988. (in Chinese). 